\title{
Opinion Article: METASTASIS AS A FAULTY RECAPITULATION OF ONTOGENY
}

Ulf R. Rapp, Institut für Medizinische Strahlenkunde und Zellforschung (MSZ), Versbacherstr. 5, 97078 Würzburg, Germany, Email: rappur@uni-wuerzburg.de

\begin{abstract}
RAF oncogenes are involved in a variety of phenotypic switch phenomena. If for example oncogenic RAF is expressed together with Myc in B lineage cells, a lineage switch to macrophages occurs at low frequency in vitro and in vivo. In addition, if RAF is expressed in type II alveolar epithelial cells slow growing lung adenomas are formed and a switch from columnar to cuboidal cells is detected in these mice upon p53 deletion. A similar switch is also seen, if ectopic Myc is present in our lung tumor mouse model. Moreover, in the liver of these mice with both oncogenes metastases are found. If E-cadherin function is impaired in our RAF-dependent lung tumor model, a switch from adenoma to adenocarcinoma occurs and genes characteristic for the early endodermal lineage are expressed. Based on these data I propose a novel model of metastasis and describe its implications. The hallmark of the model is the induction of a state of plasticity in tumor cells, which allows the reversal of differentiation to an earlier point in their ontogenic history.
\end{abstract}

\section{Introduction}

Tumor metastasis is the leading cause of cancer death and consequently a major focus of research for more than 100 years. Although basic concepts such as the "seed and soil" hypothesis (Paget, 1889) or the idea that dissemination of metastases is predominantly determined by mechanical factors (Ewing, 1928) go back a long time, a deeper understanding of the molecular details of metastasis has only been gained recently (Fidler et al., 2002; Gupta and Massague, 2006; Eccles and Welch, 2007). For example, in the wake of the discovery of "dominant" oncogenes from viruses and transformed cells and insights into their generation by gain-of-function mutations, analogous strategies to those employed for oncogene isolation were used to identify metastasis genes (Nguyen and Massague, 2007). In addition, metastasis suppressor genes blocking metastasis but not tumor formation (Steeg, 2003) and a "lung metastasis signature" with a set of genes mediating breast cancer metastasis specifically to the lung (Minn et al., 2005) have been identified. 
Aberrant gene expression patterns and altered gene functions are key features of malignant progression and growing evidence now suggests that not only genetic but also epigenetic processes are causally involved in this deregulation (Jones and Baylin, 2007). Epigenetic mechanisms have also been considered as a driving force for malignant progression in the context of epithelialmesenchymal transition (EMT). EMT describes the process of reversible transition between tissue-bound epithelial and motile mesenchymal cells and is essential for several biological processes such as neural tube and mesoderm formation or wound healing (Thiery and Sleeman, 2006). Although EMT shares many phenotypic similarities with metastatic invasion its contribution to tumor progression is still controversial (Tarin et al., 2005; Hugo et al., 2007).

Another line of evidence demonstrating the particular importance of epigenetic mechanisms for tumor progression comes from the discovery of oncogene-induced cellular plasticity (Principato et al., 1988; Nutt et al., 1999; Fedorov et al., 2003; Rawlins and Hogan, 2006; Ceteci et al., 2007). These findings form the basis for the novel concept of metastatic conversion that is presented in this article.

\section{Experimental Background}

More than 30 years ago the induction of cellular plasticity or phenotypic instability by oncogenes was discovered in studies on co-operating oncogenes such as Myc and RAF that we have found to coexist in MH2, a particularly vicious carcinoma inducing avian retrovirus (Jansen et al., 1984). Myc and RAF act synergistically (see Fig. 1 and (Fedorov et al., 2003; Dominguez-Sola et al., 2007), presumably because deleterious single oncogene effects cancel out each other in combination and because complementary steps in cell cycle progression are being activated. Moreover, both key aspects of carcinogenesis - initiation and promotion are being perpetuated in double oncogene expressing cells. Whereas Myc is associated with genomic instability (Prochownik and $\mathrm{Li}$, 2007) RAF is promoting survival and genomic stability.

The transformation of murine bone marrow cells with the J-2 retrovirus expressing $v$-raf $/ v-m y c$ oncogenes yielded clonally related mature $B$ cells and macrophages (Principato et al., 1988). Phenotypic analysis of these transformants revealed that mature myeloid cells were derived from cells with apparent B-lineage commitment and functional immunoglobulin rearrangement. This phenomenon was referred to as B-myeloid phenotypic switch and was the first evidence for the induction 
of plasticity by a RAF/Myc combination (see Fig. 2).

To investigate whether this switch is a peculiarity of the hematopoietic system or whether it pertains also to epithelial tumors a mouse model for human lung cancer was developed by our group (Kerkhoff et al., 2000). In this model, the expression of oncogenic RAF combined with the loss of the tumor suppressor p53 was shown to be associated with a switch in the phenotype of the target epithelial cell for transformation, in this case type II cuboidal pneumocytes, which turned into columnar, non-ciliated epithelial cells (Fedorov et al., 2003). Moreover, the latter population was characterized by a low level expression of ProSP-C surfactant protein $\mathrm{C}$ and of Clara cell antigen $\mathrm{CC10}$ and may represent a progenitor pool for distal lung epithelial cells (see Fig. 2).

In a third example for a switch, oncogenic RAF was combined with the loss of another tumor suppressor gene, Ecadherin, by either inducible gene ablation or by regulated expression of a dominantnegative E-cadherin (Ceteci et al., 2007). In this most recent example tumor progression to micrometastasis was accompanied with expression of genes that are not typically expressed in type II pneumocytes but are reminiscent of the ontogenetic relationship of these cells with endoderm (Cardoso and $\mathrm{Lu}, 2006)$. A key regulator for these genes is ß-catenin that became activated as a consequence of induced E-cadherin dysfunction (Ceteci et al., 2007).

Finally, in ongoing work evaluating Myc/RAF oncogene cooperation in type II lung epithelial cells we again observed a phenotypic switch from cuboidal to columnar cells as already described in the combination of RAF with p53 loss (Rapp et al., in preparation). But in this case the switch was more frequent and correlated not only with tumor progression in the lung and micrometastasis but also with emergence of large metastases at distant sites such as the liver. These combined data were merged into a novel model for tumor progression to metastasis (see Fig. 3).

\section{The Model}

Lung-targeted expression of oncogenic RAF in type II alveolar epithelial cells leads to multiple slow growing and stable adenomas consisting of mature ProSP-C surfactant protein positive cells (Kerkhoff et al., 2000). When the function of E-cadherin was impaired in these mice by genetic means, $\beta$ catenin became activated and induced the expression of genes such as CDX2 and Atoh1 that are typical for cells of the endodermal lineage (Ceteci et al., 2007). I propose that a state of plasticity is induced in these lung tumor cells. This allows the reversal of differentiation to a point in their ontogenic history, at which endodermal 
derivatives giving rise to liver (hepatoblasts) or to primordial lung are closely related and perhaps exchangeable. Upon evasion from the primary tumor site such circulating tumor cells home to the liver and switch their tissue dependence for growth from lung to liver. Our finding of micrometastases in the case of the dom.-neg. Ecadherin/RAF combination may indicate that induction of a (committed) progenitor like phenotype is not frequent or stable enough to allow circulating tumor cells to form growing colonies. For this it might be necessary to reestablish cell adhesion via cadherins in the liver.

The overall scenario is different, when Myc is introduced instead of dom.neg. E-cadherin into the RAF expressing mice. Myc also promotes the reversal of differentiation. The resulting cells still retain cell-cell contacts, yet effectively form metastases in the liver, where they form large colonies (Rapp et al., in preparation). As there is no evidence for EMT the mode of cell migration in this case may be collective migration, i.e. migration of a cell cluster that remains connected by cell-cell junctions during movement for review see (Friedl et al., 2004).

What is the phenotype of liver metastases? Considering the proposed derivation from a (progenitor) subpopulation of the targeted alveolar epithelial cells in the primary tumor in which a (liver) stem cell- like state was induced by the forced simultaneous expression of RAF and Myc oncogenes, it might have been expected that the cellular morphology differs from that in the primary tumor. However, this is clearly not the case and consequently the model postulates reversible hepatoblast mimicry that presumably is not followed by redifferentiation to cuboidal, SP-C positive, CC10 negative type II cells due to a block to differentiation at the columnar cell stage mediated by constitutive Myc expression (Fig 3, C). A similar effect is seen in p53 ablated constitutive RAF expressing type II cell derivatives in the lung (Fedorov et al., 2003).

How early are liver colonizing lung tumor derived cells detectable? The size of liver metastases that we found at 10 months in $\mathrm{Myc} / \mathrm{RAF}$ double transgenic mice suggests that lung tumor derived cells should have first arrived in the liver between 2 to 4 months of age. Consistent with this estimation is the observation that subcutaneous injection of cells derived from the primary lung tumor at this age into immune-compromised mice lead to colonies in the liver that are similar to but also different from the primary tumor in that structures related to distal bronchioles are formed in addition to foci of columnar cells (Rapp et al., in preparation). This observation suggests that the metastasizing 
columnar cells from the primary tumor have progenitor cell qualities.

In summary, the expression of plasticity inducing oncogenes in progenitor cells, such as the subpopulation of type II cells growing into foci in our RAF driven transgenic mouse model for NSCLC, induce gene expression profiles normally present in earlier progenitors thereby retracing ontogeny of the target cell. Reexpression of earlier programs in circulating tumor cells is thought to enable such cells to seed distant organs that have similar developmental roots such as liver in case of lung or colon tumor cells.

It is also conceivable that the emergence of early developmental programs in tumor cells will force such cells to leave the primary tumor site and to actively migrate to specific target organs that are developmentally related. Variations in signal intensity of plasticity inducing pathways are thought to be paralleled by reversal of dedifferentiation similar to reversal of induced plasticity in the course of tissue regeneration (Maurange et al., 2006) except if differentiation blocking oncogenes are constitutively expressed such as Myc or presumably mutant p53. Differentiation blocking oncogenes should convert reversible dedifferentiation linked to regeneration (as occurs in the lung for example after an infection with influenza virus) into a ratchet that accumulates increasingly more immature transformed progenitors with the number of rounds of challenges to regenerate.

\section{Predictions of the Model}

(1) Developmental gene expression signatures may predict targets of metastasis With respect to "metastasis signatures" in the primary tumor, the fraction of early progenitors or the size of the "cancer stem cell" compartment is predicted to be of highest prognostic value. Analyses of tumor biopsy material for early developmental markers of various derivatives of the same germ layer from which the tissue of the primary tumor originates may be particularly helpful, even if we assume that cells leave the primary tumor site simultaneously with the acquisition of metastatic ability. Another event that may signal progression to metastasis is the emergence of daughter colonies in the tissue of origin of the primary tumor.

(2) Tumor progression is reflected by stepwise changes in gene expression patterns rather than overall chaotic transcription Reversal of ontogeny as opposed to the generation of "scrambled" phenotypes by plasticity inducing oncogenes is suggesting that the earliest detectable markers belong to the most recently suppressed programs. Although the available data on sequential changes in gene expression in differentiating 
lineages of the major organ systems and in the process of transformation are limited, there is likely to be rapid progress in this area in the near future.

(3) Phenotypic alterations of metastases at secondary sites may preclude their detection It is conceivable that some metastasizing cells will not reverse dedifferentiation after selective seeding at distant sites and instead will adopt phenotype(s) of the host tissue. If this is the case a second site tumor may not be recognized as a metastasis. Genetic models allowing lineage tracing will be most useful in evaluating this possibility.

(4) Neo-formation of organ structures at secondary sites

The fate of distant metastasis may not be limited to tumor evolution and instead induce ectopic formation of near normal structures such as alveolar ducts or cysts.

(5) Lineage selector genes as novel therapeutic targets

Under the assumption that lineage selector genes are usually not key regulatory molecules in the adult novel targets for therapeutic prevention or intervention should include lineage selector genes.

(6) Genomic remodeling

The complexity of mutations in metastases versus transplantable cancer (stem) cells is not necessarily increased. It will be of interest to see whether tetraploidisation by fusion or other mechanisms is more frequently found in metastases.

(7) Plasticity induction and the ability of cancer stem cells to build niches

The plasticity that is induced in founder cells of distant metastases may also operate in the building of niches. Therefore cells making up the microenvironment of the niche such as cells of the vasculature and other support cells including cells involved in tissue remodeling may be generated by the metastatic cell and therefore share genetic alterations.

\section{Outlook}

So far the observations on reprogramming of differentiated states in tumors have been limited by two factors: use of tissue-specific promoters for oncogene expression and lack of clonality markers. More mouse experiments need to be done with lineage tracers and conditional oncogene activation/expression that is independent of tissue-restricted promoters. These studies should be extended to experiments with purified cell populations. Transplantations should include adult animals as well as blastocyst injections. Human tumor samples, primary tumors and metastases need to be analyzed similarly in xenotransplantations.

Novel targets in oncogene bearing (transitional) progenitors for chemical and 
immunological attack have to be identified. The mechanism of the oncogene-mediated differentiation block needs to be exploited for the development of differentiation inducing treatments, in addition to drugs impairing signaling pathways, which are involved in the generation of de-repressed chromatin states.

\section{Acknowledgements}

Work on tumor formation in my lab is supported by the Deutsche Krebshilfe Mildred Scheel Foundation (grant 106253) and by the DFG (Transregio 17, International Research Training Group 1141). This model was presented at the $7^{\text {th }}$ Joint Korean-German Symposium on Molecular Genetics at Seoul National University in November 2006, at the Keystone Meeting on "Host Cell Interaction and Response to the Cancer Cell" in January 2007 and at the CNIO cancer conference "Myc and the Transcriptional Control of Proliferation and Oncogenesis" in June 2007. URR thanks Ralf Schreck for help in preparing the manuscript and Fatih Ceteci, Rudolf Götz and Christiaan Karreman for critical reading of the manuscript.

\section{Literature}

Blasi, E., Mathieson, B. J., Varesio, L., Cleveland, J. L., Borchert, P. A., and Rapp, U. R. (1985). Selective immortalization of murine macrophages from fresh bone marrow by a raf/myc recombinant murine retrovirus. Nature 318, 667-670.

Cardoso, W. V., and Lu, J. (2006). Regulation of early lung morphogenesis: questions, facts and controversies. Development 133, 1611-1624.

Ceteci, F., Ceteci, S., Karreman, C., Kramer, B. W., Asan, E., Götz, R., and Rapp, U. R. (2007). Disruption of tumor cell adhesion promotes angiogenic switch and progression to micrometastasis in RAFdriven murine lung cancer. Cancer Cell 12, 145-159.

Dominguez-Sola, D., Ying, C. Y., Grandori, C., Ruggiero, L., Chen, B., Li, M., Galloway, D. A., Gu, W., Gautier, J., and Dalla-Favera, R. (2007). Nontranscriptional control of DNA replication by c-Myc. Nature 448, 445451.

Eccles, S. A., and Welch, D. R. (2007). Metastasis: recent discoveries and novel treatment strategies. Lancet 369, 17421757.

Ewing, J. (1928). Neoplastic Diseases, 6nd edn (Philadelphia: W.B. Saunders).

Fedorov, L. M., Papadopoulos, T., Tyrsin, O. Y., Twardzik, T., Goetz, R., and Rapp, U. R. (2003). Loss of p53 in craf-induced transgenic lung adenoma leads to tumor acceleration and phenotypic switch. Cancer Res 63, 2268-2277.

Fidler, I. J., Yano, S., Zhang, R. D., Fujimaki, T., and Bucana, C. D. (2002). The seed and soil hypothesis: vascularisation and brain metastases. Lancet Oncol 3, 53-57.

Friedl, P., Hegerfeldt, Y., and Tusch, M. (2004). Collective cell migration in morphogenesis and cancer. Int J Dev Biol 48, 441-449.

Gupta, G. P., and Massague, J. (2006). Cancer metastasis: building a framework. Cell 127, 679-695. 
Hugo, H., Ackland, M. L., Blick, T., Lawrence, M. G., Clements, J. A., Williams, E. D., and Thompson, E. W. (2007). Epithelial-mesenchymal and mesenchymal-epithelial transitions in carcinoma progression. J Cell Physiol.

Jansen, H. W., Lurz, R., Bister, K., Bonner, T. I., Mark, G. E., and Rapp, U. R. (1984). Homologous cell-derived oncogenes in avian carcinoma virus $\mathrm{MH} 2$ and murine sarcoma virus 3611. Nature 307, 281-284.

Jones, P. A., and Baylin, S. B. (2007). The epigenomics of cancer. Cell 128, 683692.

Kerkhoff, E., Fedorov, L. M., Siefken, R., Walter, A. O., Papadopoulos, T., and Rapp, U. R. (2000). Lung-targeted expression of the c-Raf-1 kinase in transgenic mice exposes a novel oncogenic character of the wild-type protein. Cell Growth Differ 11, 185-190.

Maurange, C., Lee, N., and Paro, R. (2006). Signaling meets chromatin during tissue regeneration in Drosophila. Curr Opin Genet Dev 16, 485-489.

Minn, A. J., Gupta, G. P., Siegel, P. M., Bos, P. D., Shu, W., Giri, D. D., Viale, A., Olshen, A. B., Gerald, W. L., and Massague, J. (2005). Genes that mediate breast cancer metastasis to lung. Nature 436, 518-524.

Nguyen, D. X., and Massague, J. (2007). Genetic determinants of cancer metastasis. Nat Rev Genet 8, 341-352.

Nutt, S. L., Heavey, B., Rolink, A. G., and Busslinger, M. (1999). Commitment to the B-lymphoid lineage depends on the transcription factor Pax5. Nature 401, 556-562.

Paget, S. (1889). The distribution of secondary growths in cancer of the breast. Lancet 1, 571-573.

Principato, M., Klinken, S. P., Cleveland, J. L., Rapp, U. R., Holmes, K. L., Pierce, J. H., and Morse, H. C., 3rd (1988). In vitro transformation of murine bone marrow cells with a v-raf/v-myc retrovirus yields clonally related mature $\mathrm{B}$ cells and macrophages. Curr Top Microbiol Immunol 141, 31-41.
Prochownik, E. V., and Li, Y. (2007). The ever expanding role for c-Myc in promoting genomic instability. Cell Cycle 6, 1024-1029.

Rapp, U. R., Bruder, J. T., and Troppmair, J. (1994). Role of the Raf signal transduction pathway in Fos/Jun regulation and determination of cell fates, In The FOS and JUN families of transcription factors, P. E. Angel, and P. A. Herrlich, eds. (Boca Raton: CRC Press), pp. 221-247.

Rapp, U. R., Cleveland, J. L., Brightman, K., Scott, A., and Ihle, J. N. (1985). Abrogation of IL-3 and IL-2 dependence by recombinant murine retroviruses expressing v-myc oncogenes. Nature 317, 434-438.

Rawlins, E. L., and Hogan, B. L. (2006). Epithelial stem cells of the lung: privileged few or opportunities for many? Development 133, 2455-2465.

Steeg, P. S. (2003). Metastasis suppressors alter the signal transduction of cancer cells. Nat Rev Cancer 3, 55-63.

Tarin, D., Thompson, E. W., and Newgreen, D. F. (2005). The fallacy of epithelial mesenchymal transition in neoplasia. Cancer Res 65, 5996-6000; discussion 6000-5991.

Thiery, J. P., and Sleeman, J. P. (2006). Complex networks orchestrate epithelialmesenchymal transitions. Nat Rev Mol Cell Biol 7, 131-142.

Wilson, A., Murphy, M. J., Oskarsson, T., Kaloulis, K., Bettess, M. D., Oser, G. M., Pasche, A. C., Knabenhans, C., Macdonald, H. R., and Trumpp, A. (2004). c-Myc controls the balance between hematopoietic stem cell selfrenewal and differentiation. Genes Dev 18, 2747-2763. 
Fig. 1: The Balance Model: Cooperation between RAF and Myc Oncogenes. The relative expression/activity levels of RAF and Myc determine the cellular response with respect to proliferation, differentiation, senescence, apoptosis or survival. The preponderance of Myc induces genomic instability in early progenitor cells and a differentiation block, which might be followed by de-differentiation and/or apoptosis. Prevalence of RAF induces differentiation, late G1 arrest, senescence and survival in late progenitor cells and differentiated cells and promotes in general genomic stability. Equilibrium of Myc and RAF in progenitor cells results in proliferation. For more details see text and (Blasi et al., 1985; Rapp et al., 1985; Rapp et al., 1994; Wilson et al., 2004).

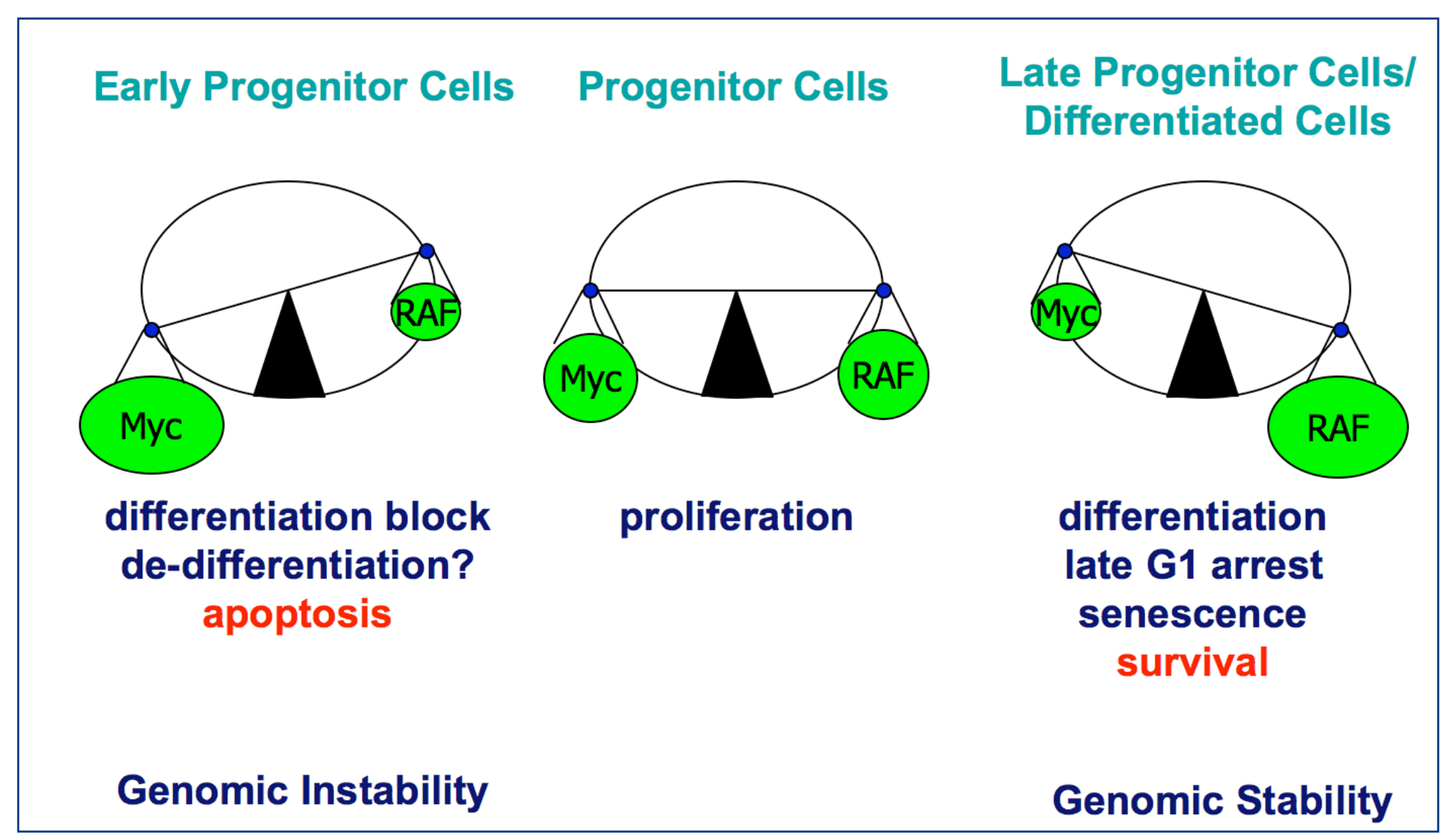


Fig. 2: In vitro and in vivo switch phenomena in hematopoietic and epithelial cell lineages involving the RAF oncogene. For more details see text.
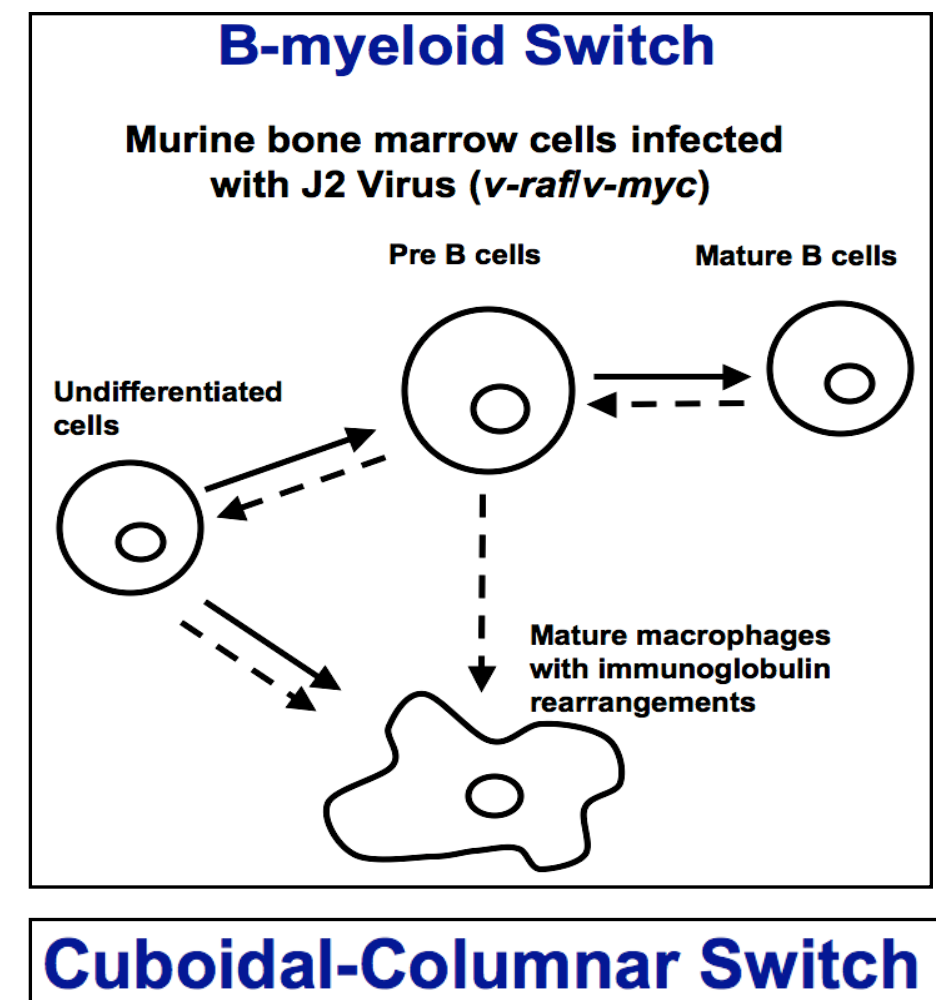

Expression of RAF and loss of p53 in type II pneumocytes of lung adenoma

Type II cuboidal cells

Columnar cells

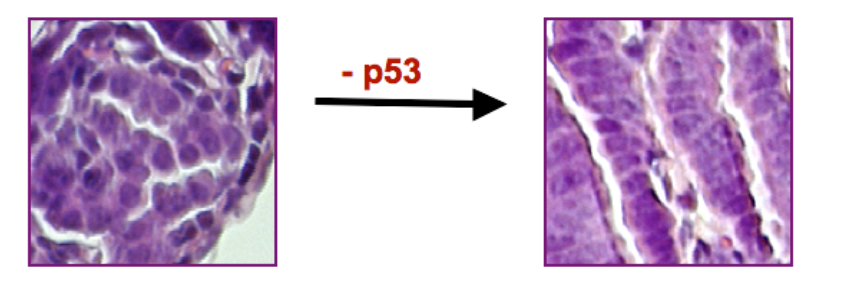

\section{Angiogenic Switch}

\section{Expression of RAF and impairment of E-cadherin} function in type II pneumocytes of lung adenoma

\section{Adenoma}

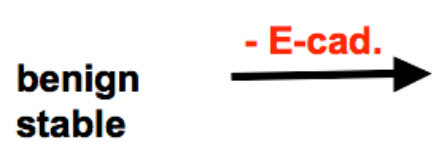

Adenocarcinoma

Neo-Angiogenesis Neo-Lymphangiogenesis Malignant Progression Invasive Growth Micrometastases

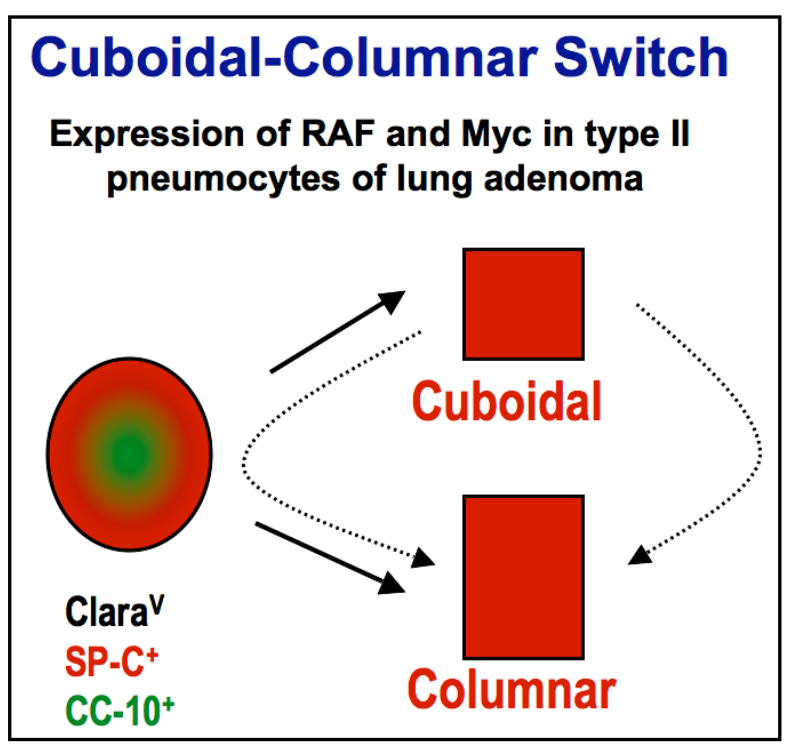


Fig. 3: Model: Metastasis - A Recapitulation of Ontogeny. (A) The lung is of endodermal origin. Following gastrulation (at E7.5 in mice) the definitive endoderm give rise to the primitive gut tube, which later forms the thyroid, thymus, trachea, lung, liver and pancreas (Cardoso and Lu, 2006). Primary lung bud and tracheal primordium formation is followed by secondary bud formation and branching morphogenesis, which results in the formation of the bronchial tree. Upon terminal differentiation the most distal region of the lung is organized into alveoli, where two types of epithelial cells are found: type I cells and cuboidal type II cells. (B) Induction of a state of plasticity, e.g. by the combination of targeted expression of oncogenic RAF in type II cells and E-cadherin impairment, allows reversal of differentiation of the type II cells to earlier points in their ontogenic history. This may lead to other lung cell types $(1,2)$ or to cells mimicking the phenotype of cells from the primitive gut tube $(3,4)$. Upon evasion from the primary tumor these cells home to tissues, which most closely resembles their phenotype, e.g. liver. Dedifferentiation is accompanied by a gain of novel potential metastatic targets and increase in malignancy of the tumor. (C) A differentiation block imposed by forced Myc expression or by p53 ablation (1) or by other factors (2) prevents redifferentiation and may further increase plasticity and heterogeneity of the transformed cell population.
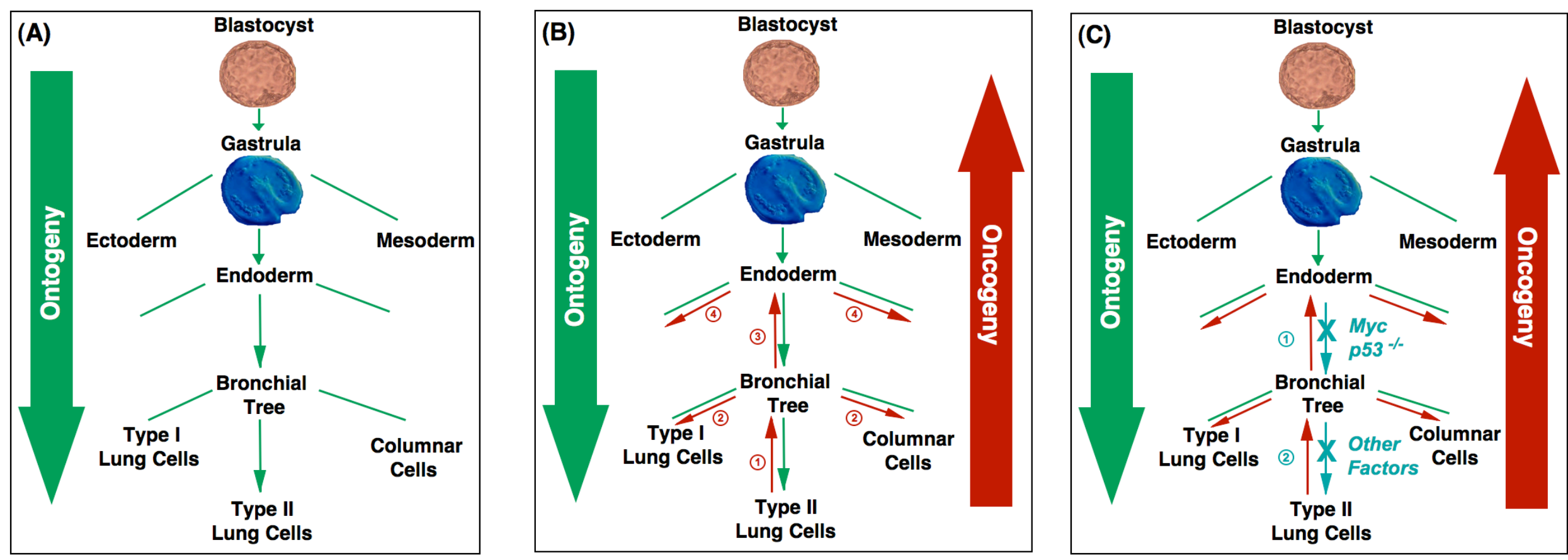